\title{
Uma Análise Experimental da Capacidade de Redes Ad Hoc Veiculares
}

\author{
Rafael dos S. Alves, Fehmi B. Abdesslem, Sávio R. Cavalcanti, Miguel Elias M. Campista, \\ Luís Henrique M. K. Costa, Marcelo G. Rubinstein, Marcelo D. de Amorim e \\ Otto Carlos M. B. Duarte
}

\begin{abstract}
Resumo-Uma classe particular de redes veiculares é aquela que utiliza equipamentos comuns, como computadores portáteis e PDAs, no interior dos veículos. Esses equipamentos estão sujeitos a condições limitadas de comunicação quando comparados com outros desenvolvidos especificamente para esse contexto. Neste artigo, as características dos enlaces formados entre nós no interior de diferentes carros são investigadas. Os resultados mostram que mesmo sem antenas externas e com equipamentos no interior do veículo, o desempenho alcançado é suficiente para executar aplicações típicas como aplicações par-a-par de transferência de arquivos. A partir dos resultados, são identificados gargalos de desempenho e formuladas recomendações de configuração e direções para trabalhos futuros.
\end{abstract}

Palavras-Chave-IEEE 802.11, Redes ad hoc, Redes ad hoc Veiculares.

Abstract-A particular class of vehicular networks is the one that includes off-the-shelf end-user equipments (e.g., laptops and PDAs) running from the interior of vehicles. These equipments are subject to limited communication conditions when compared with nodes specifically designed to this context. In this paper, we investigate through real experiments the characteristics of links formed by nodes within different cars running off-the-shelf wireless technologies. Results show that even without external antennas and using equipments within vehicles, the obtained performance is enough to run typical applications, such as file transfer in peer-to-peer applications. From our results, we identify performance issues and devise configuration recommendations and future work directions.

Keywords-IEEE 802.11, Ad hoc networks, Vehicular ad hoc networks.

\section{INTRODUÇÃO}

Redes veiculares estão entre os mais significativos e desafiadores sistemas modernos de comunicação [1]. Tanto a academia quanto a indústria estão extremamente ativos nessa área de

Fehmi B. Abdesslem e Marcelo D. de Amorim, UPMC Univ Paris 06. Sávio R. Cavalcanti, Miguel Elias M. Campista, Rafael dos S. Alves, Luís Henrique M. K. Costa e Otto Carlos M. B. Duarte, Universidade Federal do Rio de Janeiro. Marcelo G. Rubinstein, Universidade do Estado do Rio de Janeiro. E-mails: (fehmi.ben-abdesslem,marcelo.amorim)@lip6.fr, (savio,miguel,santos,luish,otto)@gta.ufrj.br, rubi@uerj.br. pesquisa e avanços fundamentais são esperados para ocorrer nos próximos anos. Um fator importante no sucesso dessas redes, como área de pesquisa, é o impacto que aplicações nesse contexto produzem no dia-a-dia das pessoas. Em particular, pode-se citar aplicações em segurança [2], entretenimento [3] e de assistência ao motorista [4].

A comunicação entre veículos pode ser feita de duas formas diferentes, em modo ad hoc puro (Vehicular Ad hoc NETwork - VANET) ou no modo infra-estruturado. No caso ad hoc, os veículos comunicam-se sem qualquer suporte externo ou elemento centralizador. No caso infra-estruturado, alguns nós estáticos são distribuídos ao longo da estrada com o objetivo de melhorar tanto a conectividade entre os nós quanto a disponibilidade de serviços. Luo et al. apresentam em detalhes as aplicações e as categorias de redes veiculares [5].

Muitos trabalhos assumem que veículos possuem antenas externas para aumentar a conectividade e/ou que um dos nós opera em modo infraestruturado [6], [7], [8], [9], [10]. Por alguns anos essa premissa não será realista. Por outro lado, é possível utilizar hoje uma configuração consistindo de equipamentos pessoais dentro dos veículos. Para os softwares existentes, não há nenhum problema para gerenciar esses nós. O problema é a maneira com que esses nós podem interagir do ponto de vista da conectividade. Neste trabalho, isso significa que os equipamentos dos usuários representam uma classe particular de nós móveis equipados com interfaces sem fio comuns, operando de dentro de uma massa metálica - o veículo. Esses nós estão sujeitos a condições de propagação mais adversas que os nós equipados com antenas externas. Mesmo assim, esse tipo de rede ad hoc veicular é viável.

Neste trabalho, uma série de testes é realizada em um cenário real para identificar os desafios de ambientes veiculares com nós internos ao veículo. São avaliados os padrões IEEE 802.11a e g, ambos usando os protocolos TCP (Transmission Control Protocol) e UDP (User Datagram Protocol). Adicionalmente, é verificado o comportamento do sistema variando-se a velocidade dos veículos e o 
tamanho dos pacotes utilizados.

As principais observações a partir dos resultados experimentais obtidos foram: $(i)$ é possível construir VANETs baseadas em nós no interior de carros que utilizam tecnologias de prateleira; (ii) a utilização de dispositivos IEEE $802.11 \mathrm{~g}$ provê vazão melhor que o IEEE 802.11a; (iii) quando o protocolo TCP é utilizado, o instante em que a conexão é requisitada é fundamental; (iv) quando o protocolo UDP é utilizado, há uma clara relação entre tamanho de pacote e vazão, a qual dependendo da velocidade do carro não é linear.

Este trabalho está organizado como se segue. $\mathrm{Na}$ Seção II, são descritos os trabalhos relacionados. Na Seção III, os equipamentos utilizados nos experimentos e os resultados obtidos são apresentados. As recomendações para o desenvolvimento de uma rede ad hoc com nós dentro de veículos são introduzidas na Seção IV. Por fim, a Seção V conclui este trabalho e apresenta os trabalhos futuros.

\section{TRABALhos RELACIONADOS}

Alguns artigos apresentam análises de medidas de comunicação entre veículos e infra-estruturas. Ott et al. [6] utilizam os protocolos UDP e TCP para transferir dados entre um carro equipado com uma antena externa e uma estação fixa conectada a um ponto de acesso IEEE 802.11b. De acordo com os autores, o emprego de antenas externas é fundamental para permitir a comunicação com o ponto de acesso. A velocidade do carro varia entre 80 e $180 \mathrm{~km} / \mathrm{h}$. Os resultados utilizando o protocolo UDP e pacotes de 1250 bytes mostram que a vazão é baixa para grandes distâncias (mais de $250 \mathrm{~m}$ até o ponto de acesso) e alcançam aproximadamente 4 Mbps quando estão na área de alcance do ponto de acesso independente da velocidade. Utilizando o protocolo TCP, a vazão apresenta uma significativa variabilidade e é menor que com o UDP.

Gass et al. [7] utilizam um cenário similar ao utilizado por Ott et al., mas sem empregar antenas externas e realizando medidas em local sem a interferência de outros pontos de acesso ou carros. A velocidade do carro varia entre 8 e $120 \mathrm{~km} / \mathrm{h}$. Já o tamanho dos pacotes UDP varia entre 50 e 1500 bytes, enquanto que com o TCP foram utilizados somente pacotes de 1500 bytes. Os resultados mostram que a máxima vazão média é obtida quando o carro está o mais próximo possível do ponto de acesso, alcançando 5,5 e 3,5 Mbps usando o TCP e o UDP, respectivamente. Os autores destacam que esse comportamento inesperado é devido aos diferentes tamanhos de pacotes utilizados durante o teste.

Bychkovsky et al. [8] utilizam um cenário completamente diferente, no qual nove carros são usa- dos. A movimentação desses carros foi observada por quase um ano. Cada carro tentava conectar-se a pontos de acesso abertos e transferir dados para um destinatário específico. As vazões máximas medidas em conexões TCP foram de aproximadamente $700 \mathrm{kbps}$.

Wellens et al. [10] testam a transferência de dados entre carros. Os autores usam o modo de operação infra-estruturado, no qual um dos carros é o ponto de acesso e o outro o cliente. Eles utilizam uma antena externa de $5 \mathrm{dBi}$ de ganho, fixa no teto do veículo. As medidas foram realizadas tanto em um cenário urbano quanto em uma auto-estrada. Os resultados mostram que a vazão útil é praticamente independente da velocidade dos carros. Os maiores fatores de impacto são a distância entre os carros, a disponibilidade de linha de visada e o algoritmo de adaptação de taxa de transmissão do IEEE 802.11.

Este trabalho é complementar aos mencionados já que um cenário diferente, com equipamentos operando no modo ad hoc, é utilizado e uma classe específica de nós é focada.

\section{MEdidAS DE CAPACIDADE ENTRE VEÍ́CULOS}

Sem perda de generalidade, uma aplicação de transferência de arquivos é considerada e a qualidade da rede é avaliada pela quantidade de dados que podem ser transferidos dentro de um enlace oportunístico. Define-se como um enlace oportunístico todo aquele formado quando veículos em movimento se encontram.

\section{A. Ambiente de Medidas}

A plataforma de testes utilizada é composta por computadores portáteis IBM T42, equipados com interfaces sem fio Linksys WPC54G (IEEE 802.11 $\mathrm{a} / \mathrm{b} / \mathrm{g}$ ) baseadas em chipset Atheros. Os computadores são levados ao colo do passageiro e antenas externas não são utilizadas. Como sistema operacional, é utilizado o Linux com kernel versão 2.6.2222-686 e driver Madwifi versão 0.9.3.3. Alguns parâmetros do algoritmo de seleção de taxa de bits chamado SampleRate padrão são ajustados. Esses ajustes foram realizados conforme sugerido em [9] e têm por objetivo aumentar a reação do algoritmo em período de rápidas mudanças. É especialmente interessante para este trabalho que o algoritmo de seleção de taxas se mantenha o maior tempo possível em altas taxas, já que o tempo de contato entre os veículos em movimento pode ser curto. Para o envio de dados e medição da qualidade do enlace, utilizamos a ferramenta de geração de tráfego Iperf em sua versão 2.0.2. 


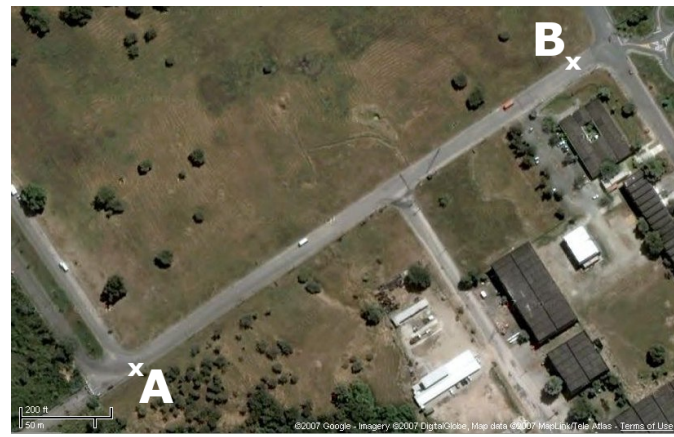

Fig. 1. Local dos experimentos (foto extraída do Google Earth).

Alguns parâmetros simples foram ajustados, antecipadamente, para evitar qualquer atraso extra devido à configuração. Para tal, os endereços IP dos portáteis foram fixados e os endereços da interface de rede dos portáteis foram adicionados a um arquivo de configuração do ARP (Address Resolution Protocol). Assim, evita-se que haja requisições a endereços de interfaces de rede de nós da rede experimental. Foram também fixados o identificador da rede (Extended Service Set ID - ESSID) e a frequiência de operação da rede, definida no padrão IEEE 802.11 por um canal de operação. Ao ajustar previamente alguns parâmetros, procura-se obter os resultados que estão perto das condições ótimas para o cenário utilizado.

Os testes foram realizados em uma rua reta de $400 \mathrm{~m}$ de extensão no campus da UFRJ (Universidade Federal do Rio de Janeiro), sob tráfego de carros leve. A Figura 1 apresenta uma imagem de satélite da rua, assim como dois pontos, $A$ e $B$, que indicam as posições de partida dos carros. Esses pontos são distantes o suficiente para que cada nó esteja fora da área de cobertura do outro. Para confirmar que os experimentos foram realizados com pouca influência externa, um mapeamento dos pontos de acesso que poderiam interferir nos resultados foi realizado. Nenhuma outra rede IEEE 802.11 opera nessa área, exceto alguns sinais fracos em outros canais detectados perto do ponto B (perto do prédio). Destacamos, entretanto, que esse segmento está fora de alcance na região em que dados são transferidos. Variamos a velocidade de ambos os carros entre 20 e $60 \mathrm{~km} / \mathrm{h}$, alterando, dessa forma, a velocidade relativa entre 40 e $120 \mathrm{~km} / \mathrm{h}$. Um dos carros utiliza o Iperf como servidor e o outro como cliente. Em ambos os casos o cliente envia dados e o servidor conta a quantidade recebida a cada $500 \mathrm{~ms}$.

Os computadores portáteis foram sincronizados, antecipadamente, utilizando o protocolo NTP (Network Time Protocol). Os carros partiam no mesmo instante. No caso em que o UDP foi o protocolo de transporte utilizado, tanto o cliente quanto o servidor foram lançados imediatamente. No caso do TCP, isso não foi possível, devido aos temporizadores de conexão do TCP. Caso o início da conexão TCP fosse realizado no começo do movimento dos carros, o temporizador expiraria antes mesmo dos carros entrarem em alcance mútuo. Como solução, o cliente se mantém enviando pacotes de sonda (pings) até que o primeiro seja respondido. Nesse momento, o Iperf é lançado. Ambos os carros movem-se pela margem direita da rua à mesma velocidade e cruzam-se aproximadamente no meio da rua. Todos os resultados utilizaram como instante de referência o momento $t=0$, no qual os carros começam a se movimentar.

Apenas os resultados com o IEEE 802.11g são apresentados, pois o uso do IEEE 802.11a possui um pior desempenho (Seção III-B). Por uma razão similar, realizamos mais análises utilizando o UDP. De fato, o protocolo UDP é mais apropriado para enlaces sem fio, pois não utiliza mecanismos de controle de congestionamento como o TCP.

Cada configuração (padrão IEEE 802.11, velocidade do veículo e tamanho de pacote) foi testada 10 vezes. Os resultados de cada experimento mostram o valor médio das 10 rodadas.

\section{B. Resultados Experimentais}

Neste trabalho o termo tempo de contato entre veículos é definido como sendo o tempo entre o primeiro e o último pacote recebido corretamente. Essa métrica é significativa para um grande número de aplicações. Em qualquer caso, é possível ter uma idéia da vazão média a partir do tempo de contato entre os carros.

A Figura 2 mostra a quantidade média de dados recebidos pelo carro que executa o servidor Iperf, quando ambos os carros estão em movimento a $20 \mathrm{~km} / \mathrm{h}$. Como mostra a Tabela I, o tempo médio de contato é de aproximadamente $35 \mathrm{~s}$. Note que esse valor de tempo não pode ser medido diretamente da figura, devido aos tempos de contato das 10 rodadas não começarem ao mesmo tempo, e porque caixas menores que 1 kbyte não são visíveis. Observa-se que o pico de vazão é de 6,4 Mbps obtido com pacotes de 1460 bytes. É importante recordar que cada ponto da curva mostra dados acumulados, em kbytes, num período de $500 \mathrm{~ms}$.

Ao aumentar a velocidade dos carros para $40 \mathrm{~km} / \mathrm{h}$, a utilização de pacotes de 1460 bytes ainda permite transferir mais dados durante o cruzamento. Todavia, como visto na Figura 3, a diferença é menor do que com $20 \mathrm{~km} / \mathrm{h}$. Nesse último caso, o tempo de contato é de aproximadamente metade 


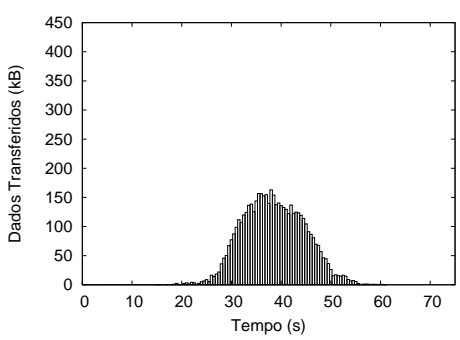

(a) Pacotes de 150 bytes.

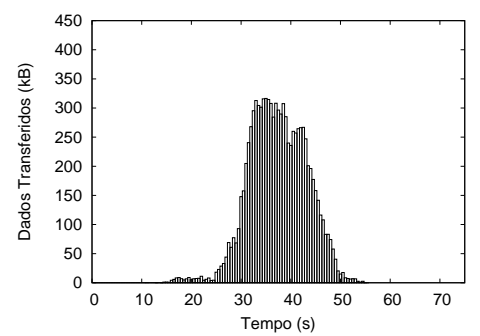

(b) Pacotes de 500 bytes.

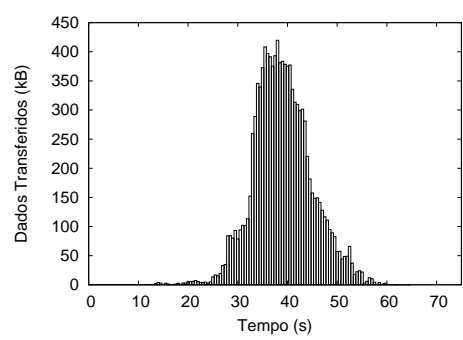

(c) Pacotes de 1460 bytes.

Fig. 2. Média de dados transferidos sobre UDP utilizando IEEE $802.11 \mathrm{~g}$ a $20 \mathrm{~km} / \mathrm{h}$.

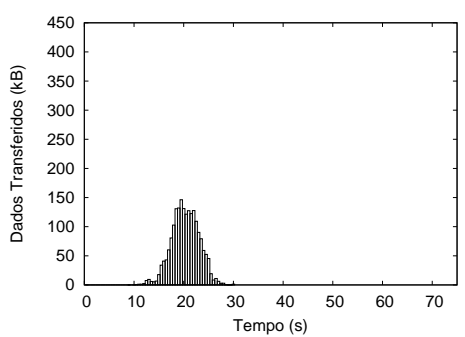

(a) Pacotes de 150 bytes.

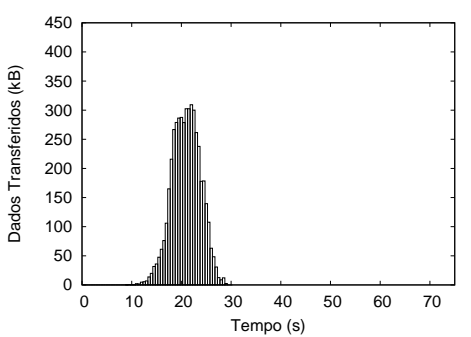

(b) Pacotes de 500 bytes.

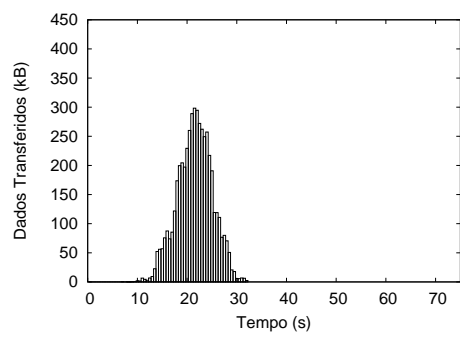

(c) Pacotes de 1460 bytes.

Fig. 3. Média de dados transferidos sobre UDP utilizando IEEE $802.11 \mathrm{~g}$ a $40 \mathrm{~km} / \mathrm{h}$.

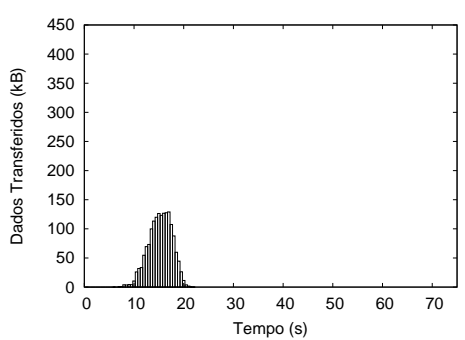

(a) Pacotes de 150 bytes.

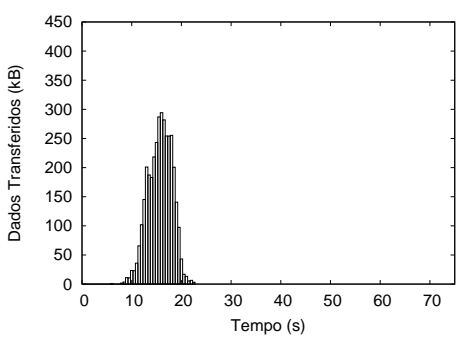

(b) Pacotes de 500 bytes.

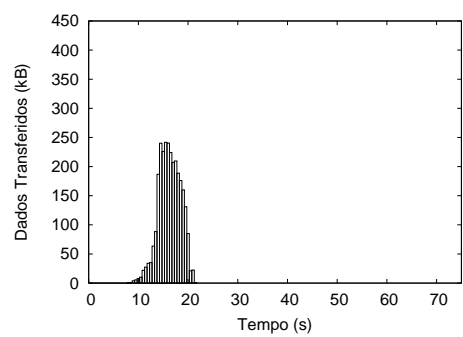

(c) Pacotes de 1460 bytes.

Fig. 4. Média de dados transferidos sobre UDP utilizando IEEE $802.11 \mathrm{~g}$ a $60 \mathrm{~km} / \mathrm{h}$.

do encontrado no caso anterior. Já a vazão máxima, ilustrada nas Figuras 3(b) e 3(c), é de aproximadamente 4,8 Mbps.

Experimentos com carros movimentando-se a $60 \mathrm{~km} / \mathrm{h}$ também foram realizados. Os resultados são mostrados na Figura 4. A Tabela I mostra o total de dados transferidos entre os dois veículos. Observa-se que quanto maior a velocidade, menor o impacto do tamanho do pacote no total de dados transferidos. No caso em que os carros se movimentam a $60 \mathrm{~km} / \mathrm{h}$, podemos observar ainda uma leve diminuição nesse valor: 3,5 Mbytes de dados foram transferidos utilizando pacotes de 500 bytes, enquanto que somente 2,8 Mbytes de dados foram transferidos utilizando pacotes de 1460 bytes. Essas medidas indicam um compromisso entre o tamanho do pacote e a velocidade. A vazão útil média das vazões úteis medidas pelo Iperf foi calculada para cada intervalo de $500 \mathrm{~ms}$. Como mostrado na Tabela I, o valor médio dessas vazões médias (calculado sobre as 10 rodadas) é aproximadamente o mesmo quando a velocidade varia e o tamanho do pacote é mantido. De fato, considerando valores de desvio padrão (denotado por $\sigma$ ), as variações de vazões úteis médias não são significativas. Logo, a redução da quantidade de dados recebidos quando a velocidade aumenta é devida principalmente a um tempo de contato mais curto.

Adicionalmente, foram realizados testes com o TCP. Os resultados foram omitidos devido a restrições de espaço e ao fraco desempenho do TCP comparado ao UDP. Comunicações utilizando o IEEE 802.11a também foram avaliadas, mas por motivos semelhantes, os resultados foram omitidos. 
TABELA I

MÉdia de DAdos TRANSFERIDOS, TEMPo DE CONTATO E VAZÃo ÚtIL UTILIZANDo o UDP E O IEEE 802.11G.

\begin{tabular}{|c|c||c|c|c|}
\hline Velocidades & $\begin{array}{c}\text { Tamanho do Pacote } \\
\text { (em bytes) }\end{array}$ & $\begin{array}{c}\text { Dados Transferidos } \\
\text { (em Mbytes) }\end{array}$ & $\begin{array}{c}\text { Tempo de Contato } \\
\text { (em segundos) }\end{array}$ & $\begin{array}{c}\text { Vazão Útil } \\
\text { (em Mbps) }\end{array}$ \\
\hline \hline \multirow{3}{*}{$\mathbf{2 0} \mathbf{~ k m / h}$} & 150 & $4,9(\sigma=0,89)$ & $35,95(\sigma=6,12)$ & $0,73(\sigma=0,12)$ \\
\cline { 2 - 5 } & 500 & $9,2(\sigma=1,23)$ & $33,95(\sigma=5,42)$ & $1,42(\sigma=0,18)$ \\
\cline { 2 - 5 } & 1460 & $10,8(\sigma=3,36)$ & $37,40(\sigma=4,99)$ & $1,60(\sigma=0,49)$ \\
\hline \multirow{3}{*}{$\mathbf{4 0} \mathbf{~ k m / h}$} & 150 & $1,9(\sigma=0,38)$ & $15,60(\sigma=3,24)$ & $0,58(\sigma=0,10)$ \\
\cline { 2 - 5 } & 500 & $4,6(\sigma=0,51)$ & $17,05(\sigma=2,91)$ & $1,33(\sigma=0,16)$ \\
\cline { 2 - 5 } & 1460 & $4,8(\sigma=1,64)$ & $16,65(\sigma=2,79)$ & $1,39(\sigma=0,53)$ \\
\hline \multirow{3}{*}{$\mathbf{6 0} \mathbf{~ k m / h}$} & 150 & $1,6(\sigma=0,41)$ & $11,70(\sigma=1,70)$ & $0,64(\sigma=0,18)$ \\
\cline { 2 - 5 } & 500 & $3,5(\sigma=1,15)$ & $11,95(\sigma=2,48)$ & $1,37(\sigma=0,48)$ \\
\cline { 2 - 5 } & 1460 & $2,8(\sigma=1,66)$ & $10,50(\sigma=1,74)$ & $1,12(\sigma=0,61)$ \\
\hline
\end{tabular}

\section{Discuss ÃO E RECOMENDAÇÕES}

De acordo com os experimentos realizados, verifica-se que a capacidade dos enlaces entre carros é suficiente para transferir alguns Mbytes. Portanto, aplicações par-a-par podem obter largura de banda suficiente para trocar arquivos como pequenos vídeos ou arquivos MP3.

Entretanto, a capacidade dos enlaces depende de uma série de parâmetros. Observa-se que quando os carros movimentam-se a altas velocidades, o emprego de pacotes pequenos pode ser mais eficiente que o emprego de pacotes grandes porque a probabilidade de erro é menor. Nota-se ainda que o IEEE 802.11g é mais adequado para redes veiculares que o IEEE 802.11a. Isso é devido à faixa de frequiências de operação utilizada pelo IEEE $802.11 \mathrm{~g}$ ser inferior à utilizada pelo IEEE $802.11 \mathrm{a}(2,4 \mathrm{GHz}$ contra $5 \mathrm{GHz})$ e, portanto, seu alcance de transmissão é maior mesmo utilizando equipamentos de prateleira. O protocolo UDP também é mais apropriado do que o TCP para o contexto analisado. Isso ocorre porque vazões mais altas são alcançadas com o UDP em enlaces com altas taxas de perda. Outra razão é que o protocolo TCP gasta muito tempo estabelecendo conexões fim-a-fim.

Baseado nos experimentos realizados, é possível recomendar a aplicação antecipada de alguns parâmetros simples para evitar atrasos extras. Dentre esses parâmetros pode-se citar: endereço IP fixo, para evitar atrasos devido ao uso de endereços dinâmicos (Dynamic Host Configuration Protocol - DHCP); ESSID e canal de operação fixos, para evitar atrasos de rastreamento; e para redes com número limitado de usuários conhecidos, endereço de interface de rede de todos os usuários preconfigurados em cada dispositivo para evitar requisições ARP. O problema de fixar esses parâmetros de forma antecipada é simples em redes pequenas, ou se alguns valores são definidos a priori (por uma autoridade central, por exemplo). Em um escopo maior, alguns parâmetros são difíceis de serem definidos antecipadamente. Nesse caso, algumas recomendações mais gerais podem ser feitas com o mesmo objetivo:

- maximizar o tempo de contato;

- evitar protocolos de gerenciamento de redes;

- evitar problemas de endereçamento de rede;

- evitar o roteamento.

A primeira recomendação pode ser alcançada reduzindo a velocidade ou aumentando a potência de transmissão de dados utilizada pelas interfaces de rede dos veículos. Já a segunda, é esperado que parâmetros como endereçamento IP, ESSID, canal de operação, algoritmo de controle de taxa sejam ajustados dinamicamente em um futuro próximo, sem a necessidade de entidades centrais. A terceira recomendação está ligada com um problema grave em redes sem fio móveis. O deslocamento dos nós não contribui com a estrutura hierárquica da Internet que é utilizada pelo protocolo IP. Assim, recomenda-se a difusão do endereço, associando placas de carros ou endereço de interface de rede ao endereço IP, ou a utilização de uma infraestrutura centralizadora, em um primeiro momento. Por fim, a última recomendação é evitar o protocolo de roteamento, visto que o descobrimento de rotas em redes muito dinâmicas é um grande desafio. Para tal, é recomendado o emprego de comunicações apenas quando o par origem-destino da comunicação estiver dentro de alcance.

A Tabela II resume as características básicas de aplicações atuais conhecidas e a adequação de cada uma para as redes veiculares, de acordo com os resultados experimentais. As aplicações distribuídas são adaptadas para esse cenário, pois estão em concordância com a arquitetura da rede. Note que qualquer aplicação DTN (Delay Tolerant Network) pode suportar altos atrasos e desconexões freqüentes [11]. Essas aplicações possuem alta aplicabilidade em ambientes veiculares, já que a rede é suscetível a freqüentes quebras de enlaces.

Conforme os experimentos deste trabalho, 
TABELA II

CARACTERÍSTICAS DE APLICAÇÕES E SUA APLICABILIDADE EM CENÁRIOS NOS QUAIS OS NÓS ESTÃo DENTRO DOS VEÍCULOS.

\begin{tabular}{|c||c|c|c|c||c|}
\hline \multirow{2}{*}{ Aplicações } & \multicolumn{4}{c||}{ Características } & Aplicabilidade \\
\cline { 2 - 6 } & $\begin{array}{c}\text { Tempo de Contato } \\
\text { Necessário }\end{array}$ & $\begin{array}{c}\text { Protocolo de } \\
\text { Transporte }\end{array}$ & $\begin{array}{c}\text { Quantidade de } \\
\text { dados transferidos }\end{array}$ & $\begin{array}{c}\text { Tipo de } \\
\text { Arquitetura }\end{array}$ & aplicável \\
\hline \hline P2P & longo & TCP ou UDP & grande & distribuída & distribuída \\
\hline Segurança & curto & UDP & pequena & distribuída & aplicável \\
\hline Assistência & curto & UDP & pequena & distribuída & aplicável \\
\hline DTN & variável & TCP ou UDP & variável & cliente-servidor & aplicável \\
\hline FTP & longo & TCP ou UDP & grande & cliente-servidor & não aplicável ainda \\
\hline HTTP & variável & TCP & variável & cliente-servidor & não aplicável ainda \\
\hline E-mail & variável & TCP & variável & cliente-servidor & não aplicável ainda \\
\hline Voz & variável & UDP & variável & cliente-servidor & não aplicável ainda \\
\hline DNS & curto & UDP & pequena & &
\end{tabular}

também pode ser recomendada a implementação ou a utilização de aplicações que tolerem perdas de pacotes, como feito pelo protocolo UFTP (UDPbased File Transfer Protocol) [12]. Isso permite a utilização do UDP como protocolo de transporte ao mesmo tempo em que se mantém a confiabilidade do TCP para a transferência de arquivos. Apesar das frequiências do IEEE 802.11a serem mais próximas das utilizadas pelo IEEE 802.11p (padrão em desenvolvimento para as redes veiculares), é recomendada também a utilização do IEEE 802.11g devido ao seu alcance maior. Finalmente, um algoritmo adaptativo deve ser utilizado para otimizar a vazão entre os nós em redes veiculares. Esse algoritmo deve reduzir ou aumentar o tamanho dos pacotes de dados dinamicamente, de acordo com a velocidade do carro. Isso poderia ser feito a partir de uma estratégia de otimização entre camadas da pilha de protocolos utilizada para a comunicação.

\section{CONCLUSÃO}

Este trabalho apresenta avaliações experimentais da capacidade de comunicação de redes ad hoc veiculares. A análise permite deduzir uma série de recomendações que podem ajudar usuários a melhorar o desempenho de suas aplicações e ajustar parâmetros apropriadamente. É importante notar que o resultado mostrado serve como ponto de referência para análises futuras de topologias mais ricas utilizando equipamentos similares.

A partir dos resultados encontrados é possível o entendimento de propriedades básicas de comunicações sobre enlaces entre veículos. Entretanto, há mais questões a serem resolvidas antes que as redes veiculares estejam completamente caracterizadas. Uma delas é a análise de cenários mais densos, sujeitos a interferências. Em trabalhos futuros, planeja-se executar aplicações reais entre nós veiculares para obter expectativas realísticas que usuários teriam de tais redes.

\section{REFERÊNCIAS}

[1] R. Morris, J. Jannotti, F. Kaashoek, J. Li e D. de Couto, "CarNet: A scalable ad hoc wireless network system", em ACM SIGOPS European Workshop, Kolding, Dinamarca, setembro de 2000

[2] W. Chen e S. Cai, "Ad hoc peer-to-peer network architecture for vehicle safety communications", IEEE Communications Magazine, vol. 43, no. 4, pp. 100-107, abril de 2005.

[3] A. Nandan, S. Das, G. Pau, M. Gerla e M. Y. Sanadidi, "Co-operative downloading in vehicular ad-hoc wireless networks", em IEEE Wireless On demand Network Systems and Services (WONS), Sankt-Moritz, Suíça, janeiro de 2005.

[4] L. Wischhof, A. Ebner, H. Rohling, M. Lott e R. Halfmann, "SOTIS: A self-organizing traffic information system", em IEEE Vehicular Technology Conference, Jeju, Coréia do Sul, pp. 2442-2446, abril de 2003.

[5] J. Luo e J.-P. Hubaux, "A survey of inter-vehicle communication", tech. rep., School of Computer and Communication Sciences, EPFL, Switzerland, 2004. Technical report IC/2004/24.

[6] J. Ott e D. Kutscher, "Drive-thru internet: IEEE 802.11b for "automobile" users", em IEEE Conference on Computer Communications (INFOCOM), Hong Kong, março de 2004.

[7] R. Gass, J. Scott e C. Diot, "Measurements of in-motion 802.11 networking", em IEEE Workshop on Mobile Computing Systems \& Applications (WMCSA), pp. 69-74, abril de 2006.

[8] V. Bychkovsky, B. Hull, A. Miu, H. Balakrishnan e S. Madden, "A measurement study of vehicular internet access using in situ wi-fi networks", em ACM International Conference on Mobile Computing and Networking (MobiCom), Los Angeles, CA, EUA, pp. 50-61, 2006.

[9] D. Hadaller, S. Keshav, T. Brecht e S. Agarwal, "Vehicular opportunistic communication under the microscope", em ACM International Conference on Mobile Systems, Applications, and Services (MobiSys), San Juan, Porto Rico, pp. 206-219, junho de 2007.

[10] M. Wellens, B. Westphal e P. Mähönen, "Performance evaluation of IEEE 802.11-based wlans in vehicular scenarios.", em IEEE Vehicular Technology Conference, Dublin, Irlanda, pp. 1167-1171, abril de 2007.

[11] K. Fall, "A delay-tolerant network architecture for challenged Internets", em ACM SIGCOMM, Karlsruhe, Alemanha, pp. 27-34, agosto de 2003.

[12] D. Bush, "UFTP - UDP based FTP with multicast". 